\title{
Validity of the Franck-Condon Principle in the Optical Spectroscopy: Optical Conductivity of the Fröhlich Polaron
}

\author{
G. De Filippis, ${ }^{1}$ V. Cataudella, ${ }^{1}$ A. S. Mishchenko, ${ }^{2,3}$ C. A. Perroni, ${ }^{4}$ and J. T. Devreese ${ }^{5}$ \\ ${ }^{1}$ Coherentia CNR-INFM and Dipartimento di Scienze Fisiche, Università di Napoli Federico II, I-80126 Napoli, Italy \\ ${ }^{2}$ CREST, Japan Science and Technology Agency (JST), AIST, 1-1-1 Higashi, Tsukuba 305-8562, Japan \\ ${ }^{3}$ RRC "Kurchatov Institute," 123182 Moscow, Russia \\ ${ }^{4}$ Institute of Solid State Research (IFF), Research Center Jülich, Jülich, D-52425, Germany \\ ${ }^{5}$ TFVS, Departement Fysica, Universiteit Antwerpen, Universiteitsplein 1, B2610 Antwerpen, Belgium
}

(Received 30 January 2006; published 6 April 2006)

\begin{abstract}
The optical absorption of the Fröhlich polaron model is obtained by an approximation-free diagrammatic Monte Carlo method and compared with two new approximate approaches that treat lattice relaxation effects in different ways. We show that: (i) a strong coupling expansion, based on the Franck-Condon principle, well describes the optical conductivity for large coupling strengths $(\alpha>10)$; (ii) a memory function formalism with phonon broadened levels reproduces the optical response for weak coupling strengths $(\alpha<6)$ taking the dynamic lattice relaxation into account. In the coupling regime $6<\alpha<10$, the optical conductivity is a rapidly changing superposition of both Franck-Condon and dynamic contributions.
\end{abstract}

DOI: 10.1103/PhysRevLett.96.136405

The Franck-Condon (FC) principle [1] and its validity have been widely discussed in studies of optical transitions in atoms, molecules [2], and solids [3]. For instance, studies on the dynamics governing color center spectra, Mössbauer spectra [4], and tunneling in polarizable media [5] have established the limits of applicability of the FC principle in solids. Generally, the FC principle means that if only one of two coupled subsystems, e.g., an electronic subsystem, is affected by an external perturbation, the second subsystem, e.g., the lattice, is not fast enough to follow the reconstruction of the electronic configuration. In the opposite limit, when the perturbation is slow or even static, the characteristic time of lattice interconfigurational coupling $\tau_{\text {ic }}$ is short enough for the lattice to follow slow changes of the electronic state dynamically. It is clear that the justification for the FC principle is the short characteristic time of the measurement process $\tau_{\mathrm{mp}} \ll \tau_{\text {ic }}$, where $\tau_{\mathrm{mp}}$ is related to the energy gap between the initial and final states $\Delta E$ through the uncertainty principle: $\tau_{\mathrm{mp}} \simeq$ $\hbar /(\Delta E)$. Then the spectroscopic response considerably depends on the value of the ratio $\tau_{\mathrm{mp}} / \tau_{\mathrm{ic}}$. For example, in mixed valence systems, where the ionic valence fluctuates between the configurations $f^{5}$ and $f^{6}$ with characteristic time $\tau_{\text {ic }} \approx 10^{-13} \mathrm{~s}$, the spectra of fast and slow experiments are dramatically different $[6,7]$. Photoemission experiments with short characteristic times $\tau_{\mathrm{mp}} \approx 10^{-16} \mathrm{~s}$ (FC regime) reveal two lines, corresponding to $f^{5}$ and $f^{6}$ states. On the other hand, slow Mössbauer isomer shift measurements with $\tau_{\mathrm{mp}} \approx 10^{-9} \mathrm{~s}$ show a single broad peak with a mean frequency between signals from pure $f^{5}$ and $f^{6}$ shells. Finally, according to the paradigm of measurement process time, magnetic neutron scattering with $\tau_{\mathrm{mp}} \approx \tau_{\text {ic }}$ revealed both coherent lines, with all subsystems dynamically adjusted, and broad incoherent rem-
PACS numbers: 71.38.Fp, 02.70.Ss, 78.30.-j

nants of strongly damped excitation of $f^{5}$ and $f^{6}$ shells [8]. Actually, the meaning and the definition of the times $\tau_{\text {ic }}$ and $\tau_{\mathrm{mp}}$ vary with the system and with the measurement process, although the spectroscopic response essentially depends on whether one of the two interacting subsystems is adjusted to the changes, induced by a measurement probe, in the other subsystem.

To investigate the interplay between the measurement process time $\tau_{\mathrm{mp}}$ and the adjustment time $\tau_{\text {ic }}$, we study in this Letter the optical conductivity (OC) of a paradigmatic model for electron-phonon ( $e$-ph) interaction: the Fröhlich model. Our aim is to investigate the $\mathrm{OC}$ from the weak to the strong coupling regime (in this model, the $e$-ph coupling strength is controlled by the dimensionless parameter $\alpha$ ) by three methods: (i) the diagrammatic Monte Carlo (DMC) method $[9,10]$, which gives numerically exact answers in all $e$-ph coupling regimes; (ii) the memory function formalism (MFF), which is able to take dynamical lattice relaxation into account; and (iii) a strong coupling expansion (SCE), which assumes the FC principle. In the coupling regime $6<\alpha<10$, we find that the OC spectrum exhibits two features with different behavior. The higherfrequency feature quickly decreases its spectral weight with increasing coupling constant, whereas the lowerfrequency feature does the opposite. Besides, the numerically exact calculations of the OC (DMC) follow the prediction of the extended MFF for $\alpha<6$, while they are in fair agreement with SCE for $\alpha>10$. We conclude that nonadiabaticity destroys the FC classification for $\alpha<10$, while the FC principle rapidly regains its validity at large coupling strengths due to the fast growth of the energy separation between the initial and final states of the optical transitions. Furthermore, both adiabatic FC and nonadiabatic dynamical excitations coexist in the intermediate $e$-ph coupling regime $6<\alpha<10$. The crossover is con- 
trolled by the adjustment time $\tau_{\text {ic }} \approx \hbar / \mathcal{D}$, set by the typical nonadiabatic energy $\mathcal{D}$ (see text below).

In the Fröhlich polaron model [11], the electron ( $\vec{r}$ and $\vec{p}$ are the position and momentum operators, respectively) is scattered by phonons $\left(a_{\vec{q}}^{\dagger}\right.$ denotes the creation operator with wave number $\vec{q}$ ) with $e$-ph interaction vertex $M_{q}=$ $i \hbar \omega_{0}\left(R_{p} 4 \pi \alpha / q^{2} V\right)^{1 / 2}$ :

$$
H=p^{2} / 2 m+\hbar \omega_{0} \sum_{\vec{q}} a_{\vec{q}}^{\dagger} a_{\vec{q}}+\sum_{\vec{q}}\left[M_{q} e^{i \vec{q} \cdot \vec{r}} a_{\vec{q}}+\text { H.c. }\right] .
$$

Here $\alpha$ is the dimensionless coupling constant, $R_{p}=$ $\left(\hbar / 2 m \omega_{0}\right)^{1 / 2}$, and $V$ is the volume of the system. The band mass of the electron $m$, Planck's constant $\hbar$, the electron charge, and the dispersionless longitudinal optical phonon frequency $\omega_{0}$ are set below to unity. Although the OC of this model attracted attention for years [12], there exists no analytic approach giving a satisfactory description for all coupling regimes. The most successful approach is that based on the Feynman path integral technique [13] (DSG), where the OC is calculated starting from the Feynman variational model (FVM) [14] for the polaron and using the path integral response formalism [15]. Subsequently, the path integral approach was rewritten in terms of the MFF [16]. These approaches are completely successful at small $e$-ph couplings and are able to identify some of the excitations at intermediate and strong $e$-ph couplings without reproducing the broad structures present in DMC data [9].

Extended memory function formalism. - In order to solve the aforementioned problem regarding the description of the OC main peak linewidth at intermediate $e$-ph couplings, we modified the DSG approach to include additional dissipation processes, whose strength is fixed by an exact sum rule. Within the MFF [17], the interaction of the charge carriers with the free phonon oscillations can be expressed in terms of the electron density-density correlation function $\chi(\vec{q}, t)=-i \theta(t)\langle\exp [i \vec{q} \cdot \vec{r}(t)] \exp [-i \vec{q} \cdot$ $\vec{r}(0)]\rangle$, which is evaluated in a direct way [16] using the FVM, where the electron is coupled via a harmonic force to a fictitious particle that simulates the phonon degrees of freedom. Within this procedure, the electron densitydensity correlation function takes the form: $\chi_{m}(\vec{q}, t)=$ $-i \theta(t) \exp \left[-i q^{2} t / 2 M\right] \exp \left[-q^{2} R\left(1-e^{-i v t}\right) / 2 M\right]$, where $R=(M-1) / v$, and $M$ (the total mass of electron and fictitious particle) and $v$ are determined variationally within the path integral approach [14]. The associated spectral function $A_{m}(\vec{q}, \omega)=-2 \operatorname{Im} \chi_{m}(\vec{q}, \omega)$ is a series of $\delta$ functions centered at $q^{2} / 2 M+n v$ ( $n$ is an integer). Here $q^{2} / 2 M$ represents the energy of the center of mass of an electron and a fictitious particle, and $v$ is the energy gap between the levels of the relative motion. To include dissipation, we introduce here a finite lifetime for the states of the relative motion, which can be considered as the result of the residual $e$-ph interaction not included into the FVM. To this end, in $\chi_{m}(\vec{q}, t)$ we replace the factor $\exp [-i v t]$ with $(1+i t / \tau)^{-v \tau}$, which leads to the replacement of $\delta$ functions by Gamma functions with mean value and variance given, respectively, by $q^{2} / 2 M+n v$ and $n v / \tau$. The parameter of dissipation $\tau$ is not an adjustable parameter but is determined by the third sum rule for $A(\vec{q}, \omega)$, which is additional to the first two that are already satisfied in the DSG model without damping. As expected, $\tau$ turns out to be of the order of $\omega_{0}^{-1}$. If broadening of the oscillator levels is neglected, $\tau \rightarrow \infty$, the DSG results $[13,16]$ are recovered. Within this latter approach $[13,16]$, the polaron OC was previously interpreted in terms of relaxed excited states transitions, FC transitions, and transitions to scattering states $[18,19]$.

Strong coupling expansion. - In the limit of strong coupling strengths, the adiabatic lattice deformation is large and the lattice kinetic energy can be regarded as a perturbation. Within the Landau and Pekar (LP) [20] approach, adiabatic lattice displacements are taken into account by a unitary transformation and the Hamiltonian is divided into two contributions $H=H_{0}+H_{I}$ : The first one describes the electron oscillation in a self-consistent quadratic potential with frequency $\omega_{1}^{\mathrm{LP}}=4 \alpha^{2} / 9 \pi$, and the second one represents the residual $e$-ph interaction. To get a quantitative estimate for the characteristic frequency of the quadratic potential, we improve the LP value considering the effects of the translational invariance and the residual interaction $H_{I}$ [21]. It turns out that the frequency $\omega_{1}=$ $\left(4 \alpha^{2} / 9 \pi-3.8\right)$ differs from that of LP $\omega_{1}^{\mathrm{LP}}$ by a constant shift 3.8. Then, starting from the Kubo formula, taking into account all multiphonon processes and neglecting recoil as well as correlation between the emission and absorption of successive phonons in all orders of the perturbation $H_{I}$, one arrives at the following expression for the real part of the OC:

$$
\operatorname{Re} \sigma(\omega)=\sigma_{0} \omega \sum_{n=0}^{\infty} \frac{e^{-\omega_{s}}}{n !}\left(\omega_{s}\right)^{n} \delta\left(\omega-\omega_{2}-n\right),
$$

resembling that expected for an exactly solvable independent oscillators model [22]. Parameters, specific for the Fröhlich polaron model, are $\omega_{2}=\omega_{1}-\omega_{s}, \sigma_{0}=\pi / 2 \omega_{1}$, and $\omega_{s}=\alpha\left(\omega_{1} / 16 \pi\right)^{1 / 2}$. The parameter $\omega_{1}$ is the FC transition energy, and $\omega_{s}$ is the energy shift due to lattice relaxation. Naturally, for large enough coupling strengths, the envelope of the Poisson distribution (2) is well described by the Gaussian

$$
\operatorname{Re} \sigma(\omega)=\omega \frac{\sigma_{0}}{\left(2 \pi \omega_{s}\right)^{1 / 2}} \exp \left\{-\frac{\left(\omega-\omega_{1}\right)^{2}}{2 \omega_{s}}\right\} .
$$

Interpretation of the DMC results. - As expected, in the weak coupling regime [Fig. 1(a)], both our extended MFF with phonon broadening and the DSG [13] are in very good agreement with the DMC data [9], showing significant improvement with respect to the weak coupling perturbation approach [23], which provides a good description of the OC spectra only for very small values of $\alpha$ [24]. For $4 \leq \alpha \leq 8$, where DSG underestimates the peak width [Figs. 1(b)-1(d)], the damping, introduced in the extended 


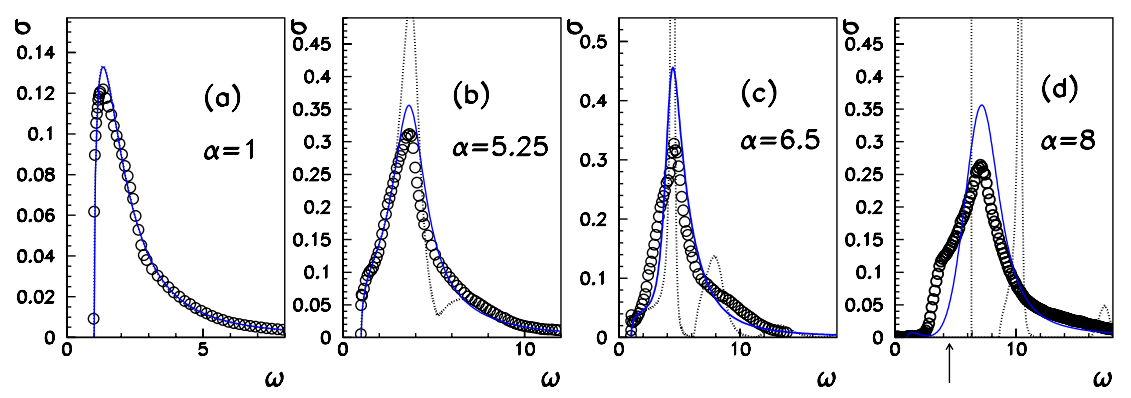

FIG. 1 (color online). Comparison of the optical conductivity calculated within the DMC method (circles), extended MFF (solid line), and DSG [13,16] (dotted line), for four different values of $\alpha$. The arrow indicates the lower-frequency feature in the DMC data.

MFF approach, becomes crucial. Results of the extended MFF are accurate for the peak energy and quite satisfactory for the peak width [Figs. 1(b)-1(d)].

However, we observe a very interesting and unexpected behavior in the coupling regime $6<\alpha<10$. Two features are present in the OC given by DMC: The position of the lower-frequency peak (or shoulder) corresponds to the predictions of the SCE [Eqs. (2) and (3)], while that for the higher-frequency peak follows the extended MFF value [Fig. 2(a)]. The higher-frequency feature rapidly decreases its intensity with increasing $\alpha$, and, at large values of $\alpha$ [Figs. 2(b) and 2(c)], the OC given by DMC is in fair agreement with the SCE results, which are strongly dominated by the FC transitions. Finally, comparing the peak and shoulder energies, obtained by $\mathrm{DMC}$, with the peak energies, given by MFF, and the FC transition energies from the SCE [Fig. 2(d)], we conclude that as $\alpha$ increases from 6 to 10 the spectral weights rapidly switch from the dynamic regime, where the lattice follows the electron motion, to the adiabatic regime dominated by FC transitions, where the nuclei are frozen in their initial configuration.

Breakdown of the FC picture. - In order to support this scenario, we present an analytical estimation of the FC breakdown based on the following arguments. The approximate adiabatic states $\chi_{i, \beta}(Q) \psi_{i}(\mathbf{r}, Q)$, where $i$ is the electronic index and $\chi_{i, \beta}(Q)$ is the eigenfunction of the lattice connected with the electron wave function $\psi_{i}(\mathbf{r}, Q)$, are not exact eigenstates of the system. These states are mixed by nondiagonal matrix elements of the nonadiabatic operator $\mathcal{D}$ and the exact eigenstates are linear combinations of the adiabatic wave functions. Being interested in the properties of transitions from the ground $(G)$ state to an excited (EX) state, whose energy corresponds to that of the OC peak, we consider mixing of only these states and express the exact wave functions $\Psi_{G \text {,EX }}(\mathbf{r}, Q)$ as linear combinations $[25,26] \Psi_{G, \mathrm{EX}}=\xi_{g, \beta}^{G, \mathrm{EX}} \chi_{g, \beta} \psi_{g}+\xi_{\mathrm{ex}, \beta^{\prime}}^{G, \mathrm{eX}} \chi_{\mathrm{ex}, \beta^{\prime}} \psi_{\mathrm{ex}}$ of the adiabatic ground state $\left(\chi_{g, \beta} \psi_{g}\right)$ and the adiabatic excited state $\left(\chi_{\mathrm{ex}, \beta^{\prime}} \psi_{\mathrm{ex}}\right)$. The superposition coefficients are determined from standard techniques $[25,26]$ where the nondiagonal matrix elements of the nonadiabatic operator [25] are expressed in terms of matrix elements of the kinetic energy operator $M$, the energy gap between the excited and ground states $\Delta E=E_{\mathrm{ex}}-E_{g}$, and the number $n_{\beta}$ of phonons in the adiabatic state:

$$
\mathcal{D}^{ \pm}=M(\Delta E)^{-1} \sqrt{n_{\beta}+1 / 2 \pm 1 / 2}+M^{2}(\Delta E)^{-2} .
$$

The extent to which the lattice can follow a transition between electronic states depends on the degree of mixing between initial and final exact eigenstates through the nonadiabatic interaction. If the states are strongly mixed, the adiabatic classification has no sense, the FC transitions have no place, and the lattice is adjusted to the change of the electronic states during the transition. In the opposite limit, the adiabatic approximation is valid and FC pro-
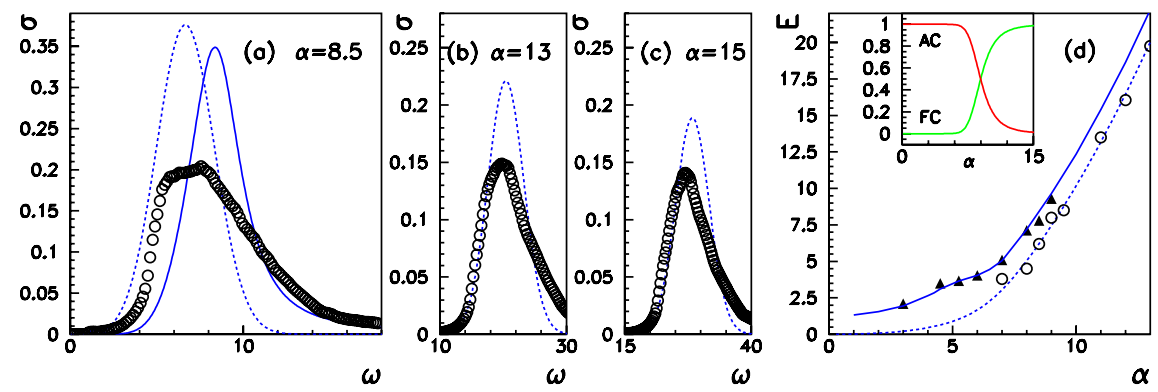

FIG. 2 (color online). (a)-(c) Comparison of the optical conductivity calculated within the DMC method (circles), the extended MFF (solid line), and the SCE (dashed line) for three different values of $\alpha$. (d) The energy of the lower- and higher-frequency features obtained by DMC (circles and triangles, respectively) compared with the FC transition energy as calculated with the SCE (dashed line) and with the energy of the peak obtained from the extended MFF (solid line). In the inset, the weights of Franck-Condon and adiabatically connected transitions are shown as a function of $\alpha$. We have used for $\eta$ the value 1.3. 
cesses dominate. An estimate for the weight of the FC component is

$$
I_{\mathrm{FC}}=1-4\left|\xi_{g, \beta}^{\mathrm{EX}} \xi_{\mathrm{ex}, \beta^{\prime}}^{G}\right|^{2},
$$

which is equal to unity in the case of zero mixing and zero in the case of maximal mixing. The weight of the adiabatically connected (AC) transition $I_{\mathrm{AC}}=1-I_{\mathrm{FC}}$ is defined accordingly. The nondiagonal matrix element $M$ is proportional to the square root of $\alpha$ with a coefficient $\eta$ of the order of unity. In the strong coupling regime, assuming that $\Delta E=\omega_{1}$ and $n_{\beta} \approx \Delta E\left(n_{\beta} \gg 1\right)$, one gets

$$
I_{\mathrm{FC}}=\left[1+4\left(\tau_{\mathrm{mp}} / \tau_{\mathrm{ic}}\right)^{2}\right]^{-1},
$$

where $\tau_{\mathrm{mp}}=1 / \Delta E$ and $\tau_{\text {ic }}=1 / D$. For $\eta$ of the order of 1 , one obtains a robust qualitative description of a rather fast switch from AC- to FC-dominated transitions, when $I_{\mathrm{FC}}$ and $I_{\mathrm{AC}}$ exchange half of their weights in the range of $\alpha$ from 7 to 10 [see inset in Fig. 2(d)]. The physical reason for such a quick change is the faster growth of the energy separation $\Delta E \sim \alpha^{2}$ compared to that of the matrix element $M \sim \alpha^{1 / 2}$. This switch has nothing to do with the self-trapping phenomenon where crossing and hybridization of the ground state and an excited state occurs. The AC-FC switch is a property of transitions between different states and is related to the choice whether the lattice can or cannot follow adiabatically the change of electronic state at the transition.

Conclusions. - Comparing numerically exact data on the optical conductivity, obtained by the diagrammatic Monte Carlo method, with results from our extended memory function approach with phonon broadened levels, which takes dynamic lattice relaxation into account, and results of the strong coupling expansion, we found that the Franck-Condon picture breaks down at $\alpha<10$. The breakdown of the Franck-Condon picture is caused by nonadiabatic mixing of initial and final states, which destroys the Franck-Condon classification scheme, and, hence, the excitation processes with dynamic adjustment of the lattice start to dominate. Finally, we find evidence for an intermediate coupling regime $6<\alpha<10$ where static and dynamic lattice responses coexist.

A. S. M. acknowledges support of RFBR Grant No. 0402-17363a and is grateful to colleagues at University of Napoli Federico II and University of Antwerpen for their hospitality.

[1] J. Franck and E. G. Dymond, Trans. Faraday Soc. 21, 536 (1926); E. U. Condon, Phys. Rev. 32, 858 (1928).
[2] D. N. Beratan and J. J. Hopfield, J. Chem. Phys. 81, 5753 (1984); X. Urbain et al., Phys. Rev. Lett. 92, 163004 (2004).

[3] M. Lax, J. Chem. Phys. 20, 1752 (1952); Y. Toyozawa, Optical Processes in Solids (Cambridge University Press, Cambridge, England, 2003).

[4] W.A. Harrison, Solid State Theory (McGraw-Hill, New York, 1970).

[5] W. B. Fowler, J. K. Rudra, M. E. Zvanut, and F. J. Feigl, Phys. Rev. B 41, 8313 (1990).

[6] D. I. Khomskii, Sov. Phys. Usp. 22, 879 (1979).

[7] C.E. T. Gonçalves da Silva and L. M. Falicov, Phys. Rev. B 13, 3948 (1976).

[8] P. A. Alekseev et al., J. Phys. Condens. Matter 7, 289 (1995); K. A. Kikoin and A. S. Mishchenko, ibid. 7, 307 (1995).

[9] A.S. Mishchenko, N. Nagaosa, N. V. Prokofev, A. Sakamoto, and B. V. Svistunov, Phys. Rev. Lett. 91, 236401 (2003).

[10] A. S. Mishchenko, N. V. Prokofev, A. Sakamoto, and B. V. Svistunov, Phys. Rev. B 62, 6317 (2000).

[11] H. Fröhlich et al., Philos. Mag. 41, 221 (1950).

[12] J.T. Devreese, in Encyclopedia of Applied Physics, edited by G. L. Trigg (VCH, New York, 1996), Vol. 14, p. 383.

[13] J. Devreese, J. De Sitter, and M. Goovaerts, Phys. Rev. B 5, 2367 (1972).

[14] R. Feynman, Phys. Rev. 97, 660 (1955).

[15] R. Feynman et al., Phys. Rev. 127, 1004 (1962).

[16] F. M. Peeters and J. T. Devreese, Phys. Rev. B 28, 6051 (1983).

[17] H. Mori, Prog. Theor. Phys. 33, 423 (1965); 34, 399 (1965); W. Götze et al., Phys. Rev. B 6, 1226 (1972).

[18] E. Kartheuser, R. Evrard, and J. Devreese, Phys. Rev. Lett. 22, 94 (1969).

[19] M. J. Goovaerts, J. M. De Sitter, and J. T. Devreese, Phys. Rev. B 7, 2639 (1973); J. T. Devreese, in Polarons in Ionic Crystals and Polar Semiconductors (North-Holland, Amsterdam, 1972).

[20] L. D. Landau and S. I. Pekar, Zh. Eksp. Teor. Fiz. 18, 419 (1948) [Sov. Phys. JETP 18, 341 (1948)].

[21] G. De Filippis et al., Eur. Phys. J. B 36, 65 (2003).

[22] G. D. Mahan, Many-Particle Physics (Plenum, New York, 1981), Chap. 4, p. 282.

[23] V. L. Gurevich et al., Fiz. Tverd. Tela (Leningrad) 4, 1252 (1962) [Sov. Phys. Solid State 4, 918 (1962)].

[24] The weak coupling limit gained some improvements by including 2-phonon processes [see W. Huybrechts and J. Devreese, Phys. Rev. B 8, 5754 (1973)], which are effectively accounted for in all orders in our approach.

[25] E. G. Brovman and Yu. Kagan, Zh. Eksp. Teor. Fiz. 52, 557 (1967) [Sov. Phys. JETP 25, 365 (1967)].

[26] K. A. Kikoin and A. S. Mishchenko, Zh. Eksp. Teor. Fiz. 104, 3810 (1993) [Sov. Phys. JETP 77, 828 (1993)]. 